\title{
Correction: Sirt3-mediated mitophagy protects tumor cells against apoptosis under hypoxia
}

\section{Aimin Qiao, ${ }^{1, *}$, Kuansong Wang 4,5,*, Yunsheng Yuan7,*, Yidi Guan'2, Xingcong Ren ${ }^{3}$, Lanya Li ${ }^{2}$, Xisha Chen ${ }^{2}$, Feng Li ${ }^{2}$, Alex F. Chen ${ }^{8,9}$, Jianda Zhou ${ }^{6}$, Jin-Ming Yang ${ }^{3}$ and Yan Cheng ${ }^{2}$}

${ }^{1}$ Center for Bioresources and Drug Discovery and School of Bioscience and Biopharmaceutics, Guangdong Pharmaceutical University, Guangzhou 510006, China

2 Department of Pharmacology, School of Pharmaceutical Sciences, Central South University, Changsha 410008, China

3 Department of Pharmacology, The Penn State Hershey Cancer Institute, The Pennsylvania State University College of Medicine and Milton S. Hershey Medical Center, Hershey, PA 17033, USA

${ }^{4}$ Department of Pathology, Xiangya Hospital, Central South University, Changsha 410008, China

${ }^{5}$ Department of Pathology, Basic Medical School, Central South University, Changsha 410008, China

${ }^{6}$ Department of Plastic Surgery, The Third Xiangya Hospital, Central South University, Changsha 410008, China

${ }^{7}$ Engineering Research Center of Cell and Therapeutic Antibody, Ministry of Education, School of Pharmacy, Shanghai Jiao Tong University, Shanghai 200240, China

${ }^{8}$ Center for Vascular and Translational Medicine, The College of Pharmacy, Central South University, Changsha 410013, China

${ }^{9}$ The Third Xiangya Hospital, Central South University, Changsha 410013, China

* These authors contributed equally to this work

Published: June 05, 2018

Copyright: Qiao et al. This is an open-access article distributed under the terms of the Creative Commons Attribution License 3.0 (CC BY 3.0), which permits unrestricted use, distribution, and reproduction in any medium, provided the original author and source are credited.

This article has been corrected: The correct figure 5A cell line T98G is given below:

The authors declare that these corrections do not change the results or conclusions of this paper.

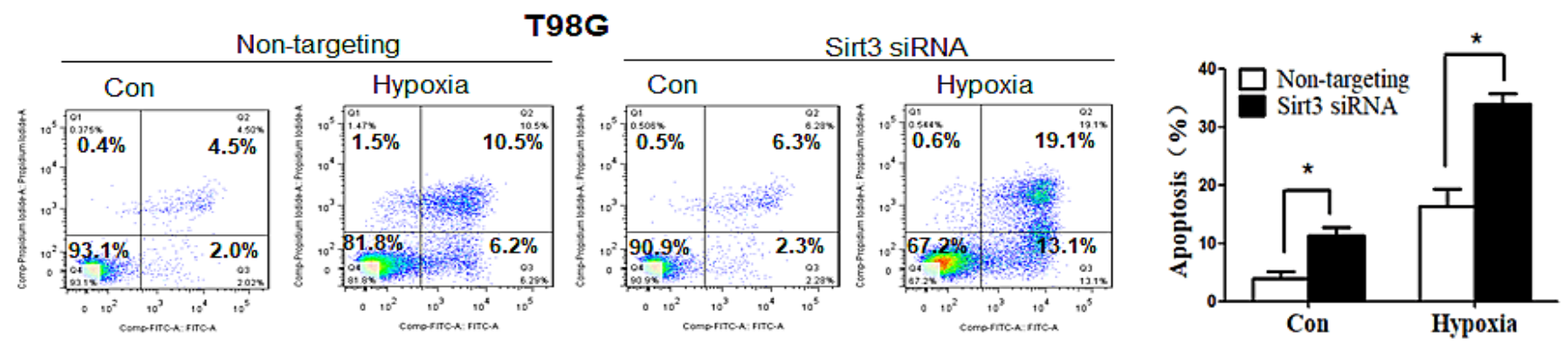

Figure 5: Suppression of Sirt3 augments the activation of apoptosis and increases the sensitivity of tumor cells to hypoxia. (A) LN229 and T98G cells were transfected with a non-targeting RNA or a siRNA targeting Sirt3, followed by hypoxia treatment for $48 \mathrm{~h}$. Apoptosis was determined by flow cytometric analyses of Annexin staining.

Original article: Oncotarget. 2016; 7:43390-43400. https://doi.org/10.18632/oncotarget.9717 